Int. J. Morphol.,

28(2):503-508, 2010.

\title{
Estudio Morfométrico y Variaciones Anatómicas del Ligamento Talocrural Lateral
}

\author{
Morphometric Study and Anatomical Variations of Lateral Talocrural Ligament
}

\author{
"Alberto Telias N.; *" René Moreno T.; "Carla Ibarra M.; "Laura Giesen F.; \\ "Bruno Capurro S.; "Paula Silva D.; "Rodrigo Sepúlveda P. \& ${ }^{\text {****** }}$ Eduardo Vega P.
}

TELIAS, N. A.; MORENO, T. R.; IBARRA, M. C.; GIESEN, F. L.; CAPURRO, S. B.; SILVA, D. P.; SEPÚLVEDA, P. R. \& VEGA, P. E. Estudio morfométrico y variaciones anatómicas del ligamento talocrural lateral. Int. J. Morphol., 28(2):503-508, 2010.

RESUMEN: Las lesiones del ligamento talofibular lateral son un problema común en traumatología. Éstas corresponden a la mayoría de los esguinces de tobillo, siendo el ligamento talofibular anterior el de mayor frecuencia, seguido del calcáneo fibular. Mediante nuestro estudio intentaremos describir la anatomía de estos ligamentos, teniendo así una herramienta para su enfrentamiento quirúrgico. Se realizó un estudio descriptivo morfométrico sobre los ligamentos talocrurales laterales, obteniéndose un promedio de sus medidas y frecuencia de variaciones anatómicas. Se disecaron 27 miembros inferiores obtenidos de amputaciones sin antecedentes de edad, sexo y morbilidad. Los ligamentos fueron medidos con un pie de metro, compás de puntas secas y regla milimetrada, expresando los resultados en milímetros. Se midió el largo(l), ancho(a), espesor(e), área de origen(ao) y área de inserción(ai). Se describieron las variaciones anatómicas que presentaron estos ligamentos. Los resultados fueron: Lig. calcáneo fibular $(l=30,3 \mathrm{~mm} ; \mathrm{a}=5,46 \mathrm{~mm} ; \mathrm{e}=2,4 \mathrm{~mm}$; $\left.\mathrm{ao}=46,17 \mathrm{~mm}^{2} ; \mathrm{ai}=66,59 \mathrm{~mm}^{2}\right)$. No presentó variaciones anatómicas. Lig. talofibular posterior $(\mathrm{l}=25,74 \mathrm{~mm} ; \mathrm{a}=6,37 \mathrm{~mm} ; \mathrm{e}=4,35 \mathrm{~mm}$; $\mathrm{ao}=40,1 \mathrm{~mm}^{2} ; \mathrm{ai}=80,19 \mathrm{~mm}^{2}$ ). En $22,2 \%$ se describe una proyección de fibras accesorias (fascículo ascendente o tibial) que nacen del origen del ligamento, dirigiéndose hacia superior para insertarse en la caraarticular de la tibia. Lig. talofibular anterior (l=20,01mm; $\mathrm{a}=10,2 \mathrm{~mm} ; \mathrm{e}=2,3 \mathrm{~mm} ; \mathrm{ao}=60,03 \mathrm{~mm}^{2} ; \mathrm{ai}=66,96 \mathrm{~mm}^{2}$ ). En $57 \%$ de los casos fue único, en $43 \%$ bifurcado y $0 \%$ trifurcado. Un $46,15 \%$ posee fibras arciformes que unen los orígenes de los ligamentos fibulotalar anterior y fibulocalcáneo. En conclusión se encontraron importantes variaciones anatómicas que no son aisladas, sino forman distintos grupos anatómicos de cada ligamento.

PALABRAS CLAVE: Ligamento lateral; Tobillo; Anatomía.

\section{INTRODUCCIÓN}

Los ligamentos talocrurales son estructuras anatómicas esenciales para la estabilidad de la articulación talocrural. Este aparato ligamentoso, debe resistir las fuerzas de tensión que se ejercen normalmente durante los movimientos del pie y en ocasiones debe soportar tensiones que pueden comprometer su indemnidad. En traumatología, las lesiones de estos ligamentos son causa de la mayoría de los esguinces de tobillo (85\%, Ferran \& Maffulli, 2006), siendo el ligamento talofibular anterior el de mayor frecuencia de lesión, seguido del calcáneo fibular (Taser et al., 2006; Kong et al., 2007).

El complejo ligamentario talocrural se compone de dos grupos, el ligamento talocrural lateral (LTCL) y el ligamento talocrural medial (LTCM). El LTCL está compuesto por tres ligamentos independientes entre si (Golanó et al., 2004):

a) Ligamento talofibular anterior (LTFA), es aplanado y cuadrilátero, encontrándose de forma único, bifurcado o trifurcado (Golanó et al.; van den Bekerom et al., 2008). Se origina en el borde anterior del maléolo fibular y se dirige anteromedialmente insertándose en el borde lateral del talus, anterior a la carilla articular destinada al maléolo fibular. En ocasiones presenta fibras arciformes, las cuales unen el LTFA en su origen maleolar con el ligamento calcáneo fibular (Golanó et al.; Sarrafian, 1993). Se dirige de lateral a medial, posterior a anterior y superior a inferior, respecto a su origen. Limita el desplazamiento anterior del talus y la flexión plantar del tobillo (van den Bekerom et al.).

* Interno de Medicina, Universidad de los Andes, Santiago de Chile.

** Interno de Medicina, Universidad de los Andes, Santiago de Chile y Servicio de Urgencia Hospital del Trabajador de Santiago, Chile.

***** Profesor de Morfología, Médico Cirujano, Universidad de los Andes, Santiago de Chile. 
b) Ligamento calcáneo fibular (LCF), grueso y de forma cordonal, cuyo origen corresponde al borde anterior del maléolo fibular, inferior al origen del LTFA. Se dirige hacia inferior y medial, y posterior, insertándose en la cara lateral del calcáneo, posterosuperior al proceso troclear de los músculos fibulares (Sarrafian, Testut \& Latarjet, 1918). No se han descrito variaciones anatómicas en la literatura sobre su morfología.

c) Ligamento talofibular posterior (LTFP), grueso y resistente (Golanó; van den Bekerom et al.), de forma trapezoidal, intracapsular y extrasinovial. Se origina en el borde medial del maléolo fibular y se dirige prácticamente paralelo al plano horizontal (Testut \& Latarjet) hacia medial, insertándose en la cara lateral y en el proceso posterolateral del talus. Puede presentar fibras ascendentes que se dirigen hacia el borde posterior de la tibia (fibras de prolongación tibial), o ligamento intermaleolar posterior (Paturet, 1951).

El objetivo de este trabajo es describir las variables macroscópicas de cada ligamento para poder entender y dilucidar las características y diferencias que determinan las propiedades del LTCL.

\section{MATERIAL Y MÉTODO}

El presente trabajo es un estudio descriptivo, realizado con muestras de miembros inferiores humanos obtenidas de amputaciones, sin conocer antecedentes de edad, sexo y comorbilidad. Fueron incluidos sólo los miembros que presentaban indemnes las zonas ligamentarias, es decir, sin ningún tipo de lesión tisular en la proximidad. No siendo incluidos los miembros que tuvieran necrosis en las zonas maleolares, calcánea u otra región cercana.

La disección se llevó a cabo por estudiantes de Medicina de la Universidad de los Andes, Santiago de Chile, en el pabellón de anatomía de la universidad, en la comuna de San Bernardo, durante el año 2009.

Los dos primeros miembros disecados por cada estudiante no fueron incluidos, siendo considerados como elementos de práctica. En total fueron utilizados 27 miembros inferiores en el estudio.

Para la disección se utilizó material quirúrgico, siguiendo un protocolo que consistió en disecar en primera instancia el tegumento, localizando el maléolo fibular, para posteriormente retirar el tejido adiposo, fascias, retináculos, músculos con sus tendones y vainas y cápsula articular, para así localizar los ligamentos (Fig.1).

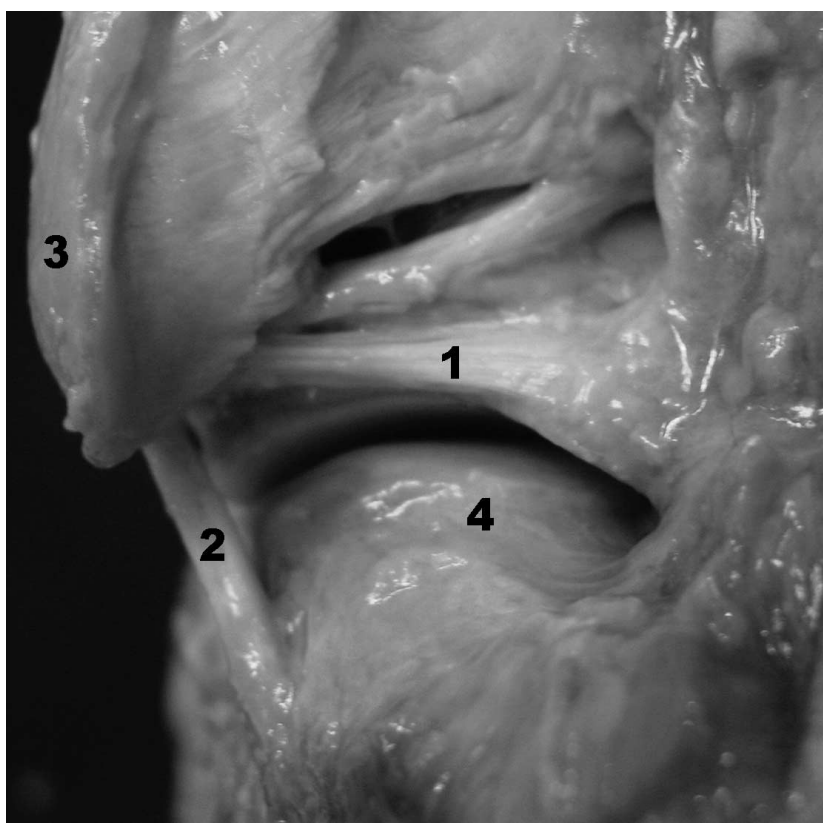

Fig. 1. Imagen obtenida del pabellón de anatomía de la Universidad de los Andes. Visión posterior de pie izquierdo. 1. LTFP, 2. LCF, 3. Fíbula, 4. Talus.

Las mediciones fueron hechas con un cáliper Mitutoyo (exactitud $0,01 \mathrm{~mm}$ ), compás geográfico y regla metálica milimetrada profesionales.

Se midió el largo, ancho, espesor, área de origen y de inserción de los ligamentos (Figs. 2, 3, 4). El largo fue medido desde el origen de las fibras centrales, hasta el punto central de la inserción. El ancho y espesor se midieron en el punto medio del ligamento. Para las áreas de origen e inser-

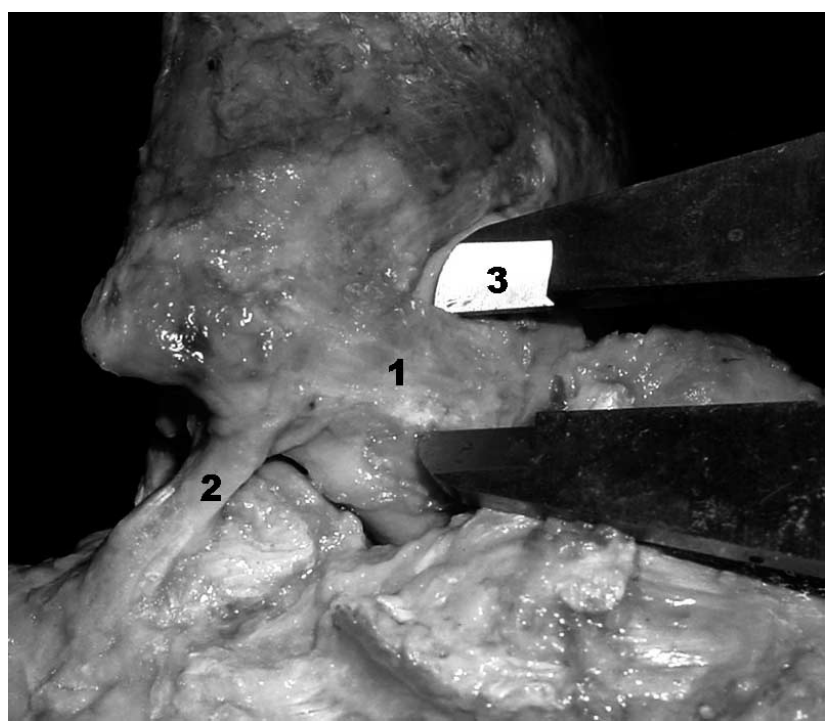

Fig. 2. Imagen obtenida del pabellón de anatomía de la Universidad de los Andes. Visión lateral de pie derecho. 1. LTFA, 2. LCF, 3. Cáliper. 


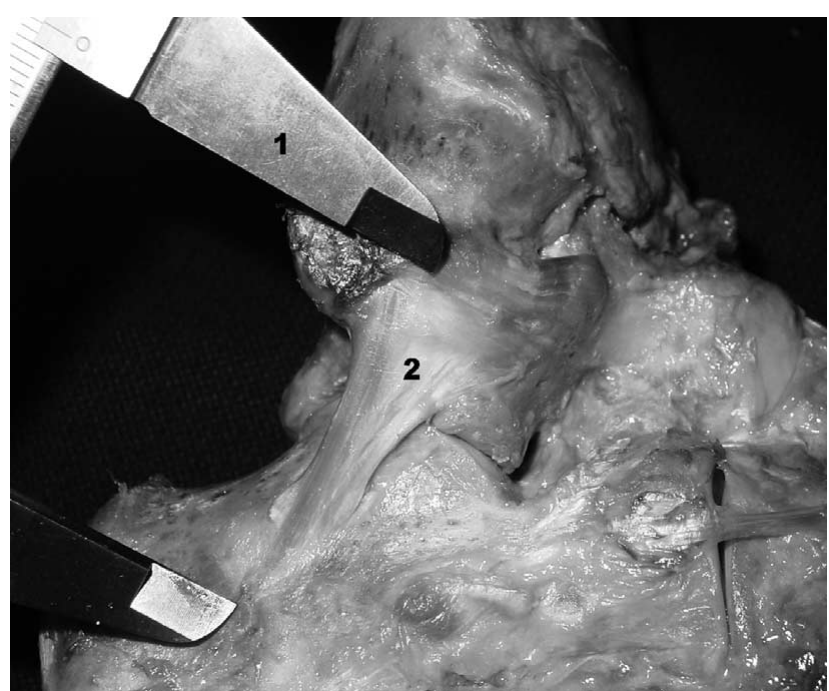

Fig. 3. Imagen obtenida del pabellón de anatomía de la Universidad de los Andes. Visión lateral de pie derecho. Medición del largo del LCF. 1. Cáliper, 2. LCF.

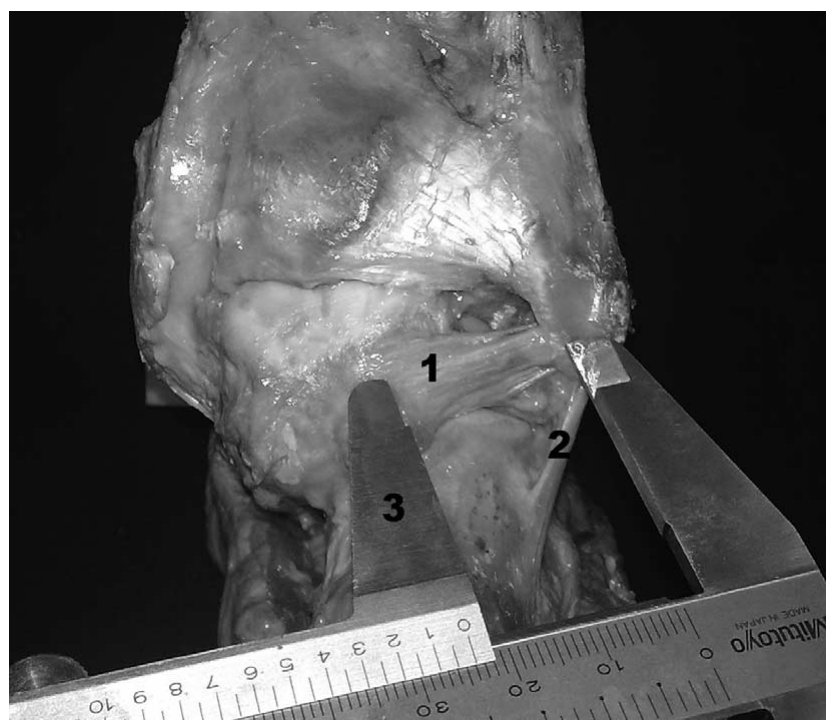

Fig. 4. Imagen obtenida del pabellón de anatomía de la Universidad de los Andes. Visión posterior de pie derecho. Medición del largo del LTFP. 1. LTFP, 2. LCF, 3. Cáliper.

ción, se utilizó la aproximación geométrica a la forma de un rectángulo, multiplicando el largo por el ancho de la superficie ligamentaria insertada en el hueso. A su vez, se describieron las variaciones anatómicas de cada ligamento.
Se utilizó el programa Microsoft Office Excel para estimar los valores promedio de cada ligamento con sus respectivas desviaciones estándar.

\section{RESULTADOS}

De las 27 muestras incluidas en el estudio 14 fueron miembros inferiores derechos (52\%) y 13 izquierdos (48\%). En cuanto al LTFA no se encontró en una muestra.

Las Tablas I y II exponen las mediciones promedios del LTFA, LCF y LTFP, y sus variaciones anatómicas respectivamente.

Los resultados promedios obtenidos del LTFA fueron: Largo: 20,01mm; Ancho: 10,2mm; Espesor: 2,3mm. El área de origen fue de $63,03 \mathrm{~mm}^{2}$, y de inserción fue de $66,96 \mathrm{~mm}^{2}$. Respecto a sus variaciones anatómicas, un $57,7 \%$ se encontró de forma única, un $42,3 \%$ bifurcado y no hubo casos de que se encontrara trifurcado. Un 46,15\% presentó fibras arciformes.

El LCF presentó como promedio las siguientes medidas: Largo: 30,3mm; Ancho: 5,46mm; Espesor: 2,4mm. $\mathrm{Su}$ área de origen promedio es de $46,17 \mathrm{~mm}^{2}$, y de inserción es $66,59 \mathrm{~mm}^{2}$. No presentó variaciones anatómicas.

El LTFP obtuvo los siguientes resultados: Largo: 25,74mm; Ancho: 6,37mm; Espesor: 4,35mm. Su área de origen es de $40,1 \mathrm{~mm}^{2}$, y de inserción es de $80,19 \mathrm{~mm}^{2}$. En un $22,2 \%$ se encontraron prolongaciones tibiales.

\section{DISCUSIÓN}

Las mediciones realizadas en este trabajo son similares a la mayoría de los estudios previamente realizados (Tabla III). En la mayoría de los estudios el LCF es el de mayor longitud, bordeando los $30 \mathrm{~mm}$ de largo. El mayor valor fue descrito por Sarrafian y Testut \& Latarjet (40mm), y el menor (19,5mm) por Milner \& Soames (1998).

Tabla I. Mediciones promedio del ligamento talocrural lateral (mm).

\begin{tabular}{lccccc}
\hline Ligamento & Largo & Ancho & Espesor & Área de origen $\left.\mathbf{( m m}^{\mathbf{2}}\right)$ & Área de inserción $\left(\mathbf{m m}^{\mathbf{2}}\right)$ \\
\hline LTFA & $20.01 \pm 4.14$ & $10.21 \pm 3.19$ & $2.3 \pm 1.01$ & $63.03 \pm 43.2$ & $66.96 \pm 36.03$ \\
LFC & $30.31 \pm 5.76$ & $5.46 \pm 2.33$ & $2.4 \pm 0.77$ & $46.17 \pm 24.27$ & $66.59 \pm 43.41$ \\
LTFP & $25.74 \pm 6.36$ & $6.37 \pm 1.8$ & $4.35 \pm 1.54$ & $40.1 \pm 28.09$ & $80.19 \pm 51.18$ \\
\hline
\end{tabular}


TELIAS, N. A.; MORENO, T. R.; IBARRA, M. C.; GIESEN, F. L.; CAPURRO, S. B.; SILVA, D. P.; SEPÚLVEDA, P. R. \& VEGA, P. E. Estudio morfométrico y variaciones anatómicas del ligamento talocrural lateral. Int. J. Morphol., 28(2):503-508, 2010.

Tabla II. Variaciones an atómicas del ligamento talocrural lateral.

\begin{tabular}{lcccc}
\hline & Único & Bifurcado & Trifurcado & Fibras arciformes \\
\hline LTFA & $57,70 \%$ & $42,30 \%$ & $0 \%$ & $46,15 \%$ \\
LFC & $*$ & & & Prolongación tibial (-) \\
\hline \multicolumn{1}{l}{ LFTP } & Prolongación tibial (+) & $22,20 \%$ \\
\hline
\end{tabular}

* No se describieron variaciones anatómicas.

El LTFP es el que lo sigue en longitud, con el mayor valor $(30 \mathrm{~mm})$ descrito por Sarrafian y el menor $(21,16 \mathrm{~mm})$ por Siegler et al. (1988).

En el caso del LTFA los diversos estudios revelan una gran variación del largo, con una longitud mayor de 24,8mm, descrita por Burks \& Morgan (1994) y menor de $13 \mathrm{~mm}$ (Milner \& Soames). Nuestro estudio se asemeja a lo descrito por Mkandawire et al. (2005) y Sarrafian principalmente.

Respecto al ancho del ligamento, no son muchos estudios los que lo cuantifican, teniendo como el LTFA el de mayor valor (de 7,2 a 11 mm), seguido del LTFP y LCF, a excepción de Sarrafian que describe al LCF más ancho que el LTFP. El ancho promedio obtenido del LCF en nuestro estudio es similar a lo descrito por la literatura, bordeando los $5 \mathrm{~mm}$. El ancho del LTFP de nuestro estudio es el mayor valor con respecto a los trabajos previos, cuyas mediciones se encuentran alrededor de los $5 \mathrm{~mm}$.

Las desviaciones estándar obtenidas en nuestro estudio son de mayor magnitud respecto a los otros. Esto pudo deberse a que los ligamentos fueron medidos por diversos investigadores previamente capacitados, pudiendo influir con mayor magnitud el error humano. También es relevante mencionar que la variación de los resultados pudo deberse a la metodología de medición e instrumentos utilizados (con su respectivo margen de error). Taser et al. utilizaron un cáliper similar al de nuestro estudio, midiendo ambos extremos del ligamento y no fibras de mayor longitud como lo realizó Burks \& Morgan. Es probable que por la metodología utilizada, los resultados del presente estudio con los de Taser et al. se asemejen, exceptuando el largo del LTFP.

Respecto a las variaciones anatómicas el LTFA, en este estudio se encontró de manera única y bifurcada, con un $57,7 \%$ y $42,3 \%$ respectivamente, sin encontrarse de forma trifurcado, en contraste con lo descrito por Milner \& Soames que en un $38 \%$ se encuentra de forma única, $50 \%$ bifurcado y $12 \%$ trifurcado. Golanó et al. y Sarrafian describen como más frecuente la forma bifurcada. Testut \& Latarjet lo describen de forma única en su tratado de anatomía, variedad más frecuente descrita en nuestro estudio.

El LCF no presentó variaciones anatómicas en su morfología tal como se describe en estudios anteriores.

En el presente estudio los ligamentos que presentan variaciones anatómicas fueron el LTFA y LTFP. Lo cual podría significar que estos ligamentos al tener más variaciones participen en menor grado en la estabilidad de la articulación talocrural. Este dato es concordante con la

Tabla III. Comparación de las dimensiones del ligamento talocrural lateral ( $\mathrm{mm})$ en diversos estudios (Adaptació n de Taser et al.).

\begin{tabular}{|c|c|c|c|c|c|c|c|c|}
\hline \multirow[t]{2}{*}{ Ligamento } & \multicolumn{2}{|c|}{ Nuestro estudio } & \multirow{2}{*}{$\frac{\text { Taser et al. }}{\text { Largo }}$} & \multicolumn{3}{|c|}{ Siegler et al. } & \multicolumn{2}{|c|}{ Mkandawire et al. } \\
\hline & Largo & Ancho & & Ancho & Largo & Ancho & Largo & Ancho \\
\hline LTFA & $20.01 \pm 4.14$ & $10.21 \pm 3.19$ & $22.37 \pm 2.5$ & $10.77 \pm 1.56$ & $17.81 \pm 3.05$ & - & $18.89 \pm 2.97$ & - \\
\hline LCF & $30.31 \pm 5.76$ & $5.46 \pm 2.33$ & $31.94 \pm 3.68$ & $4.68 \pm 1.34$ & $27.69 \pm 3.30$ & - & $35.44 \pm 6.31$ & - \\
\hline LTFP & $25.74 \pm 6.36$ & $6.37 \pm 1.8$ & $21.66 \pm 4.84$ & $5.55 \pm 1.25$ & $21.16 \pm 3.86$ & - & $27.74 \pm 3.41$ & - \\
\hline \multirow[t]{2}{*}{ Ligamento } & \multicolumn{2}{|c|}{ Milner \& Soames } & \multicolumn{2}{|c|}{ Burks \& Morgan } & \multicolumn{2}{|l|}{ Sarrafian } & \multicolumn{2}{|c|}{ Testut \& Latarjet } \\
\hline & Largo & Ancho & Largo & Ancho & Largo & Ancho & Largo & Ancho \\
\hline LTFA & $13.0 \pm 3.9$ & $11.0 \pm 3.3$ & 24,8 & 7,2 & $15,12,20$ & $8,6,5$ & - & - \\
\hline LCF & $19.5 \pm 3.9$ & $5.5 \pm 1.6$ & 35,8 & 5,3 & $30,20,30-40$ & $5,4-5,4-8$ & $30-40$ & $4-5$ \\
\hline LTFP & $23.0 \pm 7.0$ & $5.5 \pm 2.5$ & 24,1 & - & 30 & 5 & - & - \\
\hline
\end{tabular}


experiencia clínica, ya que no siempre es necesario reparar la rotura del LTFA y su reparación va a depender de la actividad que el paciente realiza.

En conclusión, las variaciones del ligamento talocrural lateral, tanto en sus medidas como en variaciones de su morfología, no son aisladas, sino pertenecen a distintos grupos de ligamentos, los cuales, en nuestro estudio son similares a los previamente descritos.

\section{AGRADECIMIENTOS}

El equipo de trabajo quiere agradecer al Dr. Juan Carlos López, al Sr. Marcos Valenzuela y al Prof. Dr. Reinhard Putz, por su apoyo, comentarios y ayuda en la realización de este trabajo.

TELIAS, N. A.; MORENO, T. R.; IBARRA, M. C.; GIESEN, F. L.; CAPURRO, S. B.; SILVA, D. P.; SEPÚLVEDA, P. R. \& VEGA, P. E. Morphometric study and anatomical variations of lateral talocrural ligament. Int. J. Morphol., 28(2):503-508, 2010.

SUMMARY: The lateral talofibular ligament injuries are a common problem in trauma. These correspond to the majority of ankle sprains, anterior talofibular ligament being the most frequent, followed by the calcaneal fibular. Through our study we will try to describe the anatomy of these ligaments, thus having a surgical tool for confrontation. A descriptive study was conducted on the morphometric lateral talocrural ligaments, resulting in an average of their measurements and frequency of anatomical variations. Were dissected 27 limbs from amputation with no history of age, sex and morbidity. The ligaments were measured with a tube foot, dry dividers and millimeter ruler, expressing the results in millimeters. We measured the length (1), width (a), thickness (e), area of origin (o) and insertion site (i). Described the anatomic variations presented these ligaments. The results were: fibulocalcaneal ligaments $(1=30.3$ $\mathrm{mm}, \mathrm{a}=5.46 \mathrm{~mm}, \mathrm{e}=2.4 \mathrm{~mm}$, ao $=46.17 \mathrm{~mm}^{2} ;$ ai $\left.=66.59 \mathrm{~mm}^{2}\right)$. No anatomic variations presented. Posterior talofibular ligament $(\mathrm{l}=$ $25.74 \mathrm{~mm}, \mathrm{a}=6.37 \mathrm{~mm}, \mathrm{e}=4.35 \mathrm{~mm}$, ao $=40.1 \mathrm{~mm}^{2}$; ai $=80.19 \mathrm{~mm}^{2}$ ). In $22.2 \%$ described a projection accessory fiber (fascicle up or fibular) arising from the origin of the ligament, superior heading to attach to the articular surface of the fibula. Anterior talofibular ligament $\left(1=20.01 \mathrm{~mm}, \mathrm{a}=10.2 \mathrm{~mm}, \mathrm{e}=2.3 \mathrm{~mm}\right.$, ao $=60.03 \mathrm{~mm}^{2} ;$ ai $\left.=66.96 \mathrm{~mm}^{2}\right)$. In $57 \%$ of cases was unique, bifurcated in $43 \%$ and $0 \%$ triple-barbed. A $46.15 \%$ have arcuate fibers connecting the origins of the anterior fibulotalar and fibulocalcaneal ligaments. In conclusion important anatomical variations were found are not isolated, but form distinct anatomical groups each ligament.

KEY WORDS: Lateral ligament, Ankle; Anatomy.

\section{REFERENCIAS BIBLIOGRÁFICAS}

Burks, R. T. \& Morgan, J. Anatomy of the lateral ankle ligaments. Am. J. Sports Med., 22(1):72-7, 1994.

Ferran, N. A. \& Maffulli, N. Epidemiology of sprains of the lateral ankle ligament complex. Foot Ankle Clin., 11(3):659-62, 2006.

Golanó, P.; Pérez-Carro, L.; Saenz, I.; Vega, J. Anatomy of ankle ligaments. Rev. Ortp. Traumatol., 48(3):33-44, 2004.

Kong, A.; Cassumbhoy, R. \& Subramaniam, R. Magnetic resonance imaging of ankle tendons and ligaments: part I - anatomy. Australas Radiol., 51(4):315-23, 2007.

Milner, C. E. \& Soames, R. W. Anatomy of the collateral ligaments of the human ankle joint. Foot Ankle Int., 19(11):757-60, 1998.
Mkandawire, C.; Ledoux, W. R.; Sangeorzan, B. J. \& Ching, R. P. Foot and ankle ligament morphometry. J. Rehabil. Res. Dev., 42(6):809-20, 2005.

Paturet, G. Traité d'antomie humaine. París, Masson, 1951.

Sarrafian, S. Anatomy of the Foot and Ankle. $2^{\text {nd }}$ Ed. Philadelphia, JB Lippincott, 1993. pp.159-217.

Siegler, S.; Block, J. \& Schneck, C. D. The mechanical characteristics of the collateral ligaments of the human ankle joint. Foot Ankle, 8(5):234-42, 1988.

Taser, F.; Shafiq, Q. \& Ebraheim, N. A. Anatomy of lateral ankle ligaments and their relationship to bony landmarks. Surg. Radiol. Anat., 28(4):391-7, 2006.

Testut, L. \& Latarjet, A. Traite d'Anatomie Humaine. $9^{\text {th }}$ Ed. Paris, Doin \& Cie, 1918. pp.711-5. 
TELIAS, N. A.; MORENO, T. R.; IBARRA, M. C.; GIESEN, F. L.; CAPURRO, S. B.; SILVA, D. P.; SEPÚLVEDA, P. R. \& VEGA, P. E. Estudio morfométrico y variaciones anatómicas del ligamento talocrural lateral. Int. J. Morphol., 28(2):503-508, 2010.

van den Bekerom, M. P.; Oostra, R. J.; Alvarez, P. G.; van Dijk, C. N. The Anatomy in Relation to Injury of the Lateral Collateral Ligaments of the Ankle: A Current Concepts Review. Clin. Anat., 21(7):619-26, 2008.

Dirección para correspondencia:

Dr. Eduardo Vega P.

Facultad de Medicina, Universidad de los Andes

San Carlos de Apoquindo 2200

Las Condes, Santiago

CHILE

Teléfono: 56-02-4129307

Email: eavegap@yahoo.com

Recibido : 21-03-2010

Aceptado: 22-04-2010 